\title{
EDITORIAL
}

\section{IN THE DECEMBER 2007 ISSUE OF CLINICS}

\author{
Mauricio Rocha-e-Silva, Editor
}

With this last issue of CLINICS in 2007 we complete our $3^{\text {rd }}$ year of existence publishing 11 papers on clinical science and 3 on basic research. Throughout this year we have lived through an increase in the number of submitted papers from approximately 209 (2005), through 260 (2006) to an expected 290-300 (2007). Published papers (not counting Editorials or Letters to the Editor) also increased, from 63 (2005) through 70 (2006) to 93 (2007). The spectrum of contributors widened, with submitted articles from outside Brazil increasing from 8 in 2005, through 12 in 2006 to 16 (Jan-Oct 2007).

In this issue, we wish to highlight a contribution by Tannuri et al, who developed an experimental model of hepatectomy (70\% removal) and liver regeneration in newborn and weaning rats, in contrast to the usual model using adult animals. Both newborn and weaning rats showed a complete recovery of liver mass within 7 days, but in the newborns this was accompanied by diffuse steatosis. They conclude that suckling and weaning rat models of partial hepatectomy are feasible and can be used for studies of liver regeneration in early life. They show interesting differences between newborn and adult liver regeneration.

Clapauch et al report on a simple doppler ultrasound test, to assess acute estradiol vascular effects on healthy and unhealthy postmenopausal women. Carotid vascular resistance (vasoconstriction) increased in unhealthy, while brachial diastolic flow (vasodilation) increased in healthy women with similar estradiol levels. This led authors to conclude that the acute estradiol test, coupled with Doppler ultrasound, seemed able to differentiate, in a simple, non-invasive manner, women with normal and abnormal endothelial function.

Vega et al describe the profile of cases of maternal death resulting from disorders in pregnancy of arterial hypertension in the city of São Paulo (1995-1999). They identified

Hospital das Clínicas, São Paulo University Medical School - São Paulo/ SP, Brazil.

Email: mrsilva36@hcnet.usp.br
609 cases of obstetric maternal death and conclude that complications of arterial hypertension are responsible for these high rates of pregnancy-related deaths. Quality prenatal care and appropriate monitoring of the hypertensive pregnant patient during and after delivery are stressed as important measures for a better control of this condition and are essential to reduce disorders in pregnancy.

Souza et al determined the frequency of anti-endothelial cell antibodies (AECA) in patients with Behçet's Disease and analyzed whether these antibodies exert any influence on clinical presentation and/or activity of the disease. They conclude that IgG-AECA might be a marker of more severe lesions in patients with Behçet's Disease based on the higher frequency of previous central nervous system manifestations in patients who presently display circulating anti-endothelial cell antibodies.

Moreira et al analyzed the performance of Brazilian medical residents and specialist trainees in radiology and diagnostic imaging in the In-Training Examination for Radiology and Diagnostic Imaging applied by the Brazilian College of Radiology. They conclude that residents' performance was better than the trainees' in the majority of the subspecialties, and suggest that the exam provides a system for evaluating future specialists, identify the centers that need to revise the teaching method, as well as search for regional differences in radiological training.

Crippa et al describe results of 8 cases of rectourethral fistula treated by York-Mason technique for recto-urethral fistulas and conclude that the performance of routine colostomy and cystostomy is unnecessary because the surgical correction of such fistulae using the technique described by York-Mason permits easy access, reduces the surgical and the hospitalization time, and presents low complication and morbidity rates.

Cardoso da Silva et al compared the measurements of contrast sensitivity at distance in patients submitted to penetrating keratoplasty versus patients submitted to deep anterior lamellar keratoplasty for keratoconus treatment, and conclude that the contrast sensitivity was similar among the 
subjects submitted to penetrating keratoplasty and to deep anterior lamellar keratoplasty for keratoconus treatment.

Vieira et al evaluated the role played by specific antibodies in lower respiratory tract infection caused by respiratory syncytial virus in infants, through aprospective study carried out win 192 patients; types and genotypes of Respiratory Syncytial Virus causing respiratory tract infection in infants were identified and the association of subtypespecific serum antibodies with the occurrence of infection was determined to evaluate the presence of subtype-specific antibodies in the infants' mothers and their association with the profile of the childrens' serum antibodies. Although the prevalence of subtype-specific antibodies was elevated, these antibodies did not provide protection independently of virus type/genotype. The similarity in the profiles of subtype-specific antibodies presented by the mothers and their children was compatible with transplacental passage.

Greve et al correlated body mass index and postural balance in 40 males, aged $26 \pm 5 \mathrm{yrs}$, through functional stability tests using the Biodex Balance System (stability evaluation protocol level 2, which allows an inclination of up to $20^{\circ}$ in the horizontal plane in all directions) in order to compare with body mass index, showing that high body mass index demands more displacements to maintain postural balance.

Saraiva et al investigated the consistency of optical coherence tomography on foveal thickness, foveal volume, and macular volume measurements in 20 patients with and 20 without diffuse diabetic macular edema, found a good index of consistency even considering patients with unstable staring, and conclude that optical coherence tomography is a consistent method for diabetic subjects with diffuse macular edema.

Rocha e Silva et al compared midterm patency of radial artery grafts for coronary obstruction in elective and nonelective through 64-Slice Computed Tomography in 47 patients (23 elective) with critical coronary artery stenosis, and conclude that Radial Artery grafts can be used in both elective and non-elective patients with excellent results.

Carvalho et al studied the importance of NB84, synaptophysin and AgNOR explored the quantitative association of these factors with diagnosis and outcome as well as the association between NB84 and AgNOR and other tumor and stromal factors in peripheral neuroblastic tumors from 28 patients. They found that AgNOR, NB84 and synaptophysin reached statistical significance as prognostic indicators, concluding that determination of NB84 and synaptophysin are useful tools for the diagnosis of peripheral neuroblastic tumors, and that the association of the evaluation of AgNOR expression by the tumor cells may provide important contributions to the prognostic evaluation and management approach of the patients.

Doria et al evaluated the effect of intertrochanteric osteotomy on the proximal femur of rabbits: assessment with power doppler sonography and scintigraphy, and state that results warrant further investigation in determining the value of different imaging modalities to assess pathologic changes following hip surgery. Power Doppler sonography presented higher accuracy for discrimination of vascular fractional areas and scintigraphy, for discrimination of number of vessel/area unit.

Mattar et al studied the effect of a salt overload combined with administration of the NO inhibitor, N-nitro-Larginine methyl ester (NAME) on the development of renal injury in rats. And found that that treatment with a high salt diet and NAME predisposes to the development of hypertension and renal injury upon salt overload, characterizing a new model of chronic nephropathy. The response to mycophenolate mofetil or losartan, but not hydralazine, suggest a key role of inflammatory rather than hemodynamic factors.

We publish 2 review articles, by Younes et al on antitumoral and antibacterial drugs extracted from Brazilian plants and by Leão et al, on ulcerative lesions of the mouth. We also publish 5 case reports. 